\title{
Stability Analysis on the Polymorphic Compound of Thiourea Derivatives: 1,3-Bis(1-(4-Methylphenyl)Ethyl)Thiourea
}

\author{
Nur Najwa-Alyani Mohd Nabil, Nor Ain Fathihah Abdullah and Lee Sin Ang* \\ Faculty of Applied Sciences, Universiti Teknologi MARA, Perlis Branch, \\ 02600 Arau, Perlis, Malaysia
}

*Corresponding author: anglee631@perlis.uitm.edu.my

Published online: 25 February 2018

To cite this article: Najwa-Alyani, M. N. N., Abdullah, N. A. F. \& Ang, L. S. (2018). Stability analysis on the polymorphic compound of thiourea derivatives: 1,3-bis(1-(4methylphenyl)ethyl)thiourea. J. Phys. Sci., 29(Supp. 1), 17-23, https://doi.org/10.21315/ jps2018.29.s1.3

To link to this article: https://doi.org/10.21315/jps2018.29.s1.3

\begin{abstract}
Polymorphism is the ability of a compound to form more than one crystalline form in the solid state. Compounds having this ability would display different stabilities for different forms, hence affecting its applicability. Symmetrically substituted thiourea derivatives have been shown to be polymorphic due to the flexibility of the $C-N$ bond. One of this type of compounds, known as 1,3-bis(1-(4-methylphenyl)ethyl)thiourea, is studied on its stabilities and ability to exist in different forms. Since the molecule of this compound was experimentally found to exist in trans-trans configuration, the stability of the cis-trans and cis-cis configurations are assessed using theoretical calculations. Rotations along the $C-N$ bonds are performed using Gaussian 09 suite program. Level of method B3LYP, complemented by basis set DEF2-TZVP, is used. The stability of the molecules in different configurations is then studied in terms of its interactions using reduced density gradient. Apart from the original trans-trans configuration, with the difference of $0.024 \mathrm{eV}$, cis-trans configuration are also possible to exist. Next possible configuration is cis-cis with relative energy $1.8412 \mathrm{eV}$. Both the non-covalent $\mathrm{CH} \cdots H N$ and $\mathrm{CH} \cdots \mathrm{S}$ interactions are found to provide stabilising effect in the cis-trans and trans-trans configurations.
\end{abstract}

Keywords: Polymorphism, thiourea derivatives, density functional theory, topology analysis, non-covalent interactions.

\section{INTRODUCTION}

Polymorphism is a phenomenon where a compound can exist in different configurations. Its unexpected and unavoided occurrences gained interest from various industries. ${ }^{1}$ Since different forms lead to different properties, the different 
forms would affect their roles in industries. ${ }^{2}$ In this report, the focus is on 1,3-bis(1(4-methylphenyl)ethyl)thiourea, $\mathrm{C}_{19} \mathrm{H}_{24} \mathrm{~N}_{2} \mathrm{~S}_{1}$ which is a thiourea derivatives compound known as HAMFIL with ((methylphenyl)ethyl) group attached at the terminal two $\mathrm{N}$ atoms. ${ }^{3}$ The HAMFIL is like other thiourea derivatives, exhibiting potent inhibiting characteristics that will prevent a particular chemical reaction, or reduce the activity of a particular reactant or catalyst. HAMFIL exists with total symmetry with respect to its substituents where the bond, angle and torsion angle are the same for both sides of the molecule, as shown in Figure 1. The stabilities and ability to exist in different forms of groups are studied. The stability of the cistrans and cis-cis configuration are assessed using theoretical calculations.

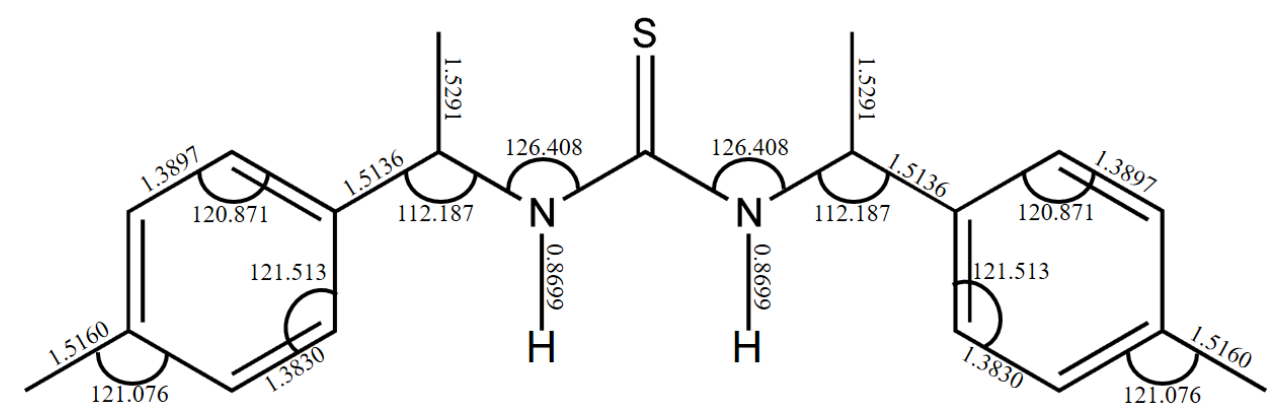

Figure 1: Molecular structure of HAMFIL and its bond lengths and angles. Only certain parameter included to indicate the total symmetry of both substituents of HAMFIL. Bond lengths are in $\AA$ and angles in ${ }^{\circ}$.

The reduced density gradient (RDG) is used in this study to show the non-covalent interactions (NCI) in compound. NCI was used to study the stability of the polymorph and interaction in complexes. ${ }^{4,5}$ The RDG, $s$ is defined as:

$$
s=\frac{1}{2\left(3 \pi^{2}\right)^{\frac{1}{3}}} \frac{|\nabla \rho|}{\rho^{\frac{4}{3}}}
$$

where $\nabla \rho$ is the gradient of the electron density. Since $s$ is dependent on $|\nabla \rho| / \rho^{4 / 3}$, a plot of $s$ vs $|\nabla \rho| / \rho^{4 / 3}$ enables the identification of weak interaction in the molecular system. The $\rho(\mathrm{r})^{4 / 3}$ term approaches zero with higher decay rate compared to $\nabla \rho$. $s$ will be high when further from the nuclei and approaches small value when closer to the nuclei.

\section{EXPERIMENTAL}

HAMFIL originally existed in trans-trans configuration as shown in Figure 2. Trans is configuration when hydrogen is opposite to the sulphur in the thiourea 
moiety. Rotation at one side of thiourea is labelled as side A while another one as side B. For dimer calculations, side of each molecules are labelled as in Figure 2. For this research, all combinations are considered and labelled as combination of $\mathrm{AC}, \mathrm{AD}, \mathrm{BC}$ and BD. For cis-cis configuration, both substituents of both molecules are rotated to cis configuration.

Single point calculations are carried out on the different configurations of the systems of interest, either single molecule or dimer. The dimer system, together with the rotations on the substituents, is shown in Figure 2.

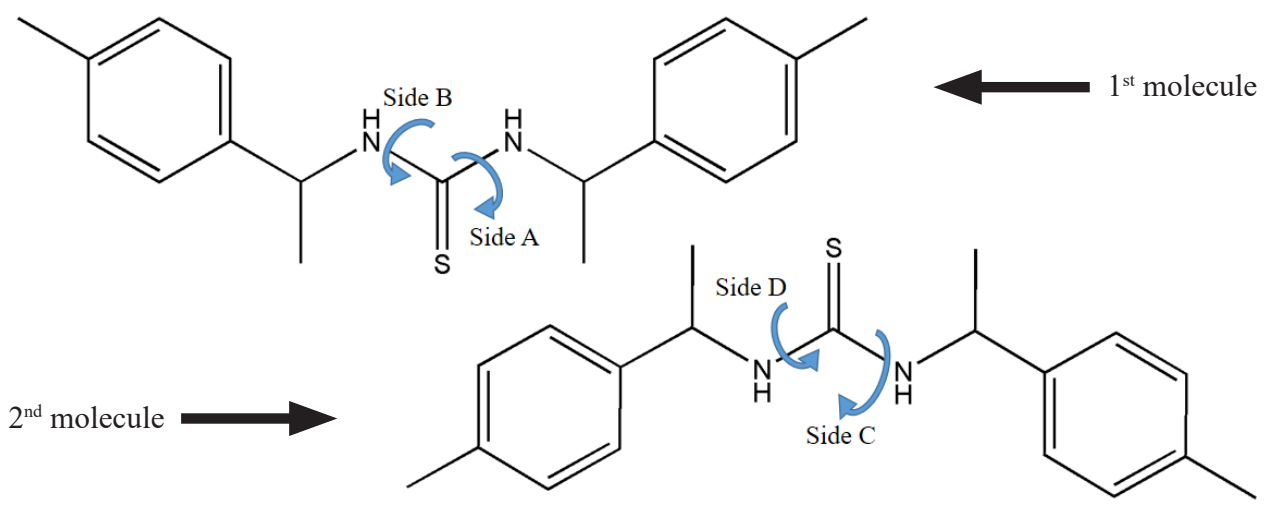

Figure 2: The structure of the dimer of HAMFIL with sides labelled as A, B, C and D.

The calculations are performed using the Gaussian 09 software at the B3LYP with the DEF2-TZVP basis set. The correctional scheme of D3-BJ and gCP are included in this study. The total energy is then given as:

$$
E_{\mathrm{total}}=E_{\mathrm{SCF}}+E_{\mathrm{D} 3-\mathrm{BJ}}+E_{\mathrm{gCP}}
$$

where $E_{\mathrm{SCF}}$ is self-consistent field energy, $E_{\mathrm{D} 3 \text {-BJ }}$ is dispersion correction and $E_{\mathrm{gCP}}$ is counterpoise correction. The total energy of different configurations are compared to the one from the trans-trans configuration.

The NCI of different configuration are performed using the Multiwfn. The results are shown through the visual molecular dynamics (VMD). NCI-RDG isosurface of a compound is coloured according to the corresponding values of $\operatorname{sign}\left(\lambda_{2}\right) \rho$ (shown in Figure 3). It is an indicator of the strength of interactions. For this study, we are using the Blue-Green-Red (BGR) scheme where blue indicates bonding interaction, green of $\mathrm{vdW}$ and red indicates non-bonding interactions. The cutoff used for the RDG, $s(r)$, is 0.3 au and the colour scale is $-0.04<\rho<0.01$. 


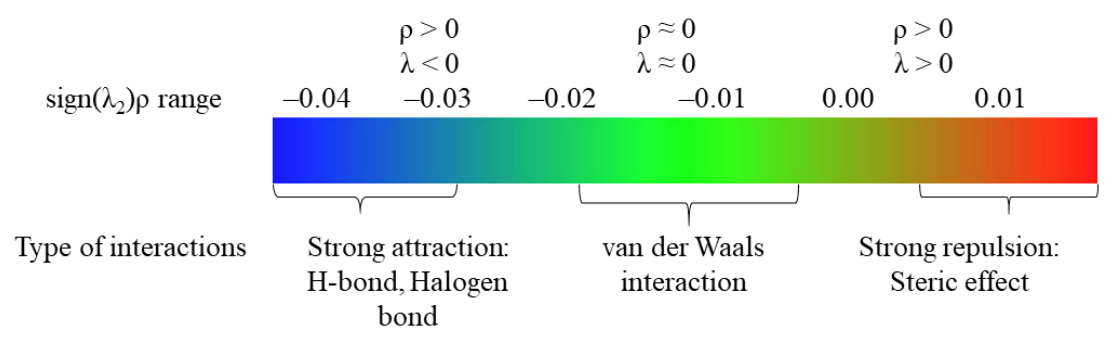

Figure 3: Colour range $\left(-0.04<\operatorname{sign}\left(\lambda_{2}\right) \rho<0.02 \mathrm{au}\right)$ of the non-covalent interactions.

\section{RESULTS AND DISCUSSION}

Table 1 shows that for the 1-molecule system, the cis-trans configuration is more stable than the original configuration of trans-trans, as the relative energy is negative. Since it is a symmetric molecule, rotation for both side (side A and B) of the molecule produced the same relative energy of $-0.0243 \mathrm{eV}$. The cis-cis configuration is less stable with a relative energy of $1.8412 \mathrm{eV}$. In this configuration, the small distance between the two hydrogens of $\mathrm{CH}($ side $\mathrm{A}) \cdots \mathrm{HC}$ (side $\mathrm{B}$ ) has a distance of $0.9548 \AA$, and creates a pseudo 7-membered ring. The ring produces repulsion at the centre of the ring, and lowered the stability of the whole system. Graphical explanation will be given later in the discussion of NCI plot.

In the case of dimer, Table 1 also shows that the cis-trans configuration is more stable with relative energy of $-0.0298 \mathrm{eV}$ for $\mathrm{BC}$ configuration. In the $\mathrm{BC}$ configuration, the first and second molecules have the $\mathrm{B}$ and $\mathrm{C}$ sides rotated to the cis configuration, while keeping A and D sides fixed. Cis-trans configurations of $\mathrm{AC}, \mathrm{AD}$ and $\mathrm{BD}$ are less stable compared to the trans-trans configuration, since they have higher positive relative energy than the one in trans-trans configuration. Higher relative energy shows that compound have less favourable intermolecular interaction compare to the trans-trans configuration. ${ }^{6}$ Cis-cis configuration also shows similar condition where the relative energy higher than trans-trans configuration with $5.8879 \mathrm{eV}$. Hence energetically, while cis-trans is shown to be able to exist, cis-cis is not a preferable configuration for HAMFIL.

Table 1: The relative energy from different configuration of HAMFIL. The capital letters for the cis-trans configuration of dimer are explained in the text.

\begin{tabular}{|c|c|c|c|c|c|}
\hline & \multicolumn{5}{|c|}{ Relative energy $(\mathrm{eV})$} \\
\hline System & Trans-trans & & Cis-trans & & Cis-cis \\
\hline 1-Molecule & 0.000 & & -0.0243 & & 1.8412 \\
\hline Dimer & 0.000 & $0.1040(\mathrm{AC})$ & $0.2574(\mathrm{AD})-0.0298(\mathrm{BC})$ & $0.1413(\mathrm{BD})$ & 5.8879 \\
\hline
\end{tabular}


The NCI plot of the trans-trans configuration shows similar isosurfaces on both of its substituents (Figure 4(a)) due to the compound having total symmetrical substituents. The dual colour isosurface of green-red at $\mathrm{CH}^{\cdots \cdots} \mathrm{HC}$ and $\mathrm{CH}^{\cdots \cdots} \mathrm{HN}$ interactions are from the combination of the van der Waals (vdW) and steric repulsion interactions of the atoms. ${ }^{5,7}$ Less steric repulsion exists at the greenyellow isosurface of $\mathrm{CH}^{\cdots} \mathrm{S}$ interaction is due to increment in their distance to $3.2546 \AA$, compare to the green-red isosurface (2.6513 $\AA$ ). The red-bullet shapes, common in the ring compound, is due to high steric repulsion. ${ }^{5}$

The NCI surfaces for cis-trans configuration are similar either rotation of side A or B. Thus, only one rotation's side are shown in Figure 4(b). This can be attributed to the total symmetry of the side substituents. Comparing the NCI plots for cis-trans and trans-trans configurations, isosurfaces for both configurations are similar. However, the $\mathrm{CH}^{\cdots} \cdot \mathrm{S}$ interaction in trans configuration is replaced with the $\mathrm{CH} \cdots \mathrm{HN}$ interaction in the cis configuration. The difference in NCI plot shows that the energy difference between these configurations are affected by the different interaction of $\mathrm{CH}^{\cdots} \cdot \mathrm{S}$ and $\mathrm{CH} \cdots \mathrm{HN}$. It also shows that the $\mathrm{CH}^{\cdots} \cdot \mathrm{HN}$ has stronger interaction compared to the $\mathrm{CH} \cdots \mathrm{S}$ interaction. This is due to the shorter distance of the former $(1.829 \AA)$ than the latter $(2.651 \AA)$.

For cis-cis configuration (Figure 4 (c)), the red-bullet isosurface is visual at the $\mathrm{H}-\mathrm{C} \cdots \mathrm{C}-\mathrm{H}$ ring closure replacing the $\mathrm{CH} \cdots \mathrm{S}$ interaction in trans-trans configuration. Red-bullet isosurface appears in ring-like interactions due to density overlap in the central part of the ring closure. ${ }^{5}$ This indicates that the lesser stability of cis-cis configuration is due to the existence of the repulsive interaction at this ring closure.
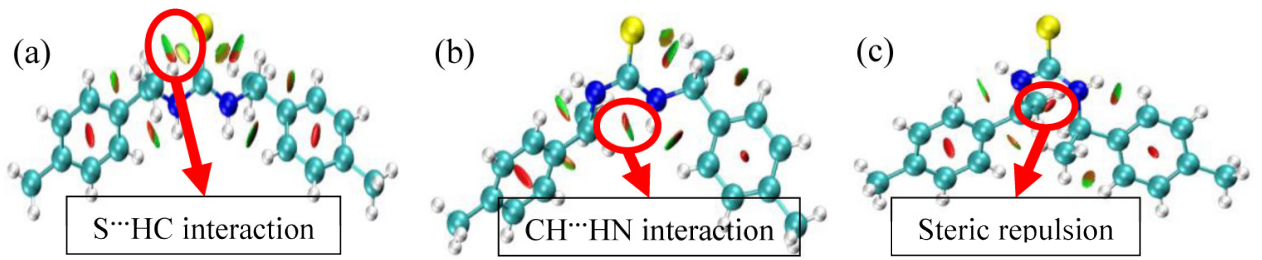

Figure 4: The NCI plot of the 1-molecule HAMFIL in (a) trans-trans, (b) cis-trans and (c) cis-cis configurations.

For the trans-trans configuration of dimer system (Figure 5(a)), NCI isosurfaces of the individual molecules are similar to the 1-molecule system. The $\mathrm{CH} \cdots \mathrm{S}$, $\mathrm{CH} \cdots \mathrm{HN}$ and $\mathrm{CH} \cdots \mathrm{HC}$ interactions of the first and second molecules have similar $\mathrm{NCI}$ isosurfaces at the specific places. Based on this observation, the difference in energies can be said to have been resulted from the interaction between the molecules in the case of dimer. The vdW interaction is shown exist between the substituents. 
For cis-trans configuration, similar NCI isosurfaces appear intramolecularly (Figure 5(b), (c) and (d)). However, the vdW interaction between molecules exists in different strengths can be ranked as $\mathrm{BC}>\mathrm{AC}>\mathrm{BD}>\mathrm{AD}$. The strengths are similar compared to the relative energy results. $\mathrm{AD}$ is not shown here due to having only slight $\mathrm{vdW}$ isosurfaces. Thus, the interaction between molecules affects the stability of dimer compound. For most energetically stable configuration which is cis-trans $\mathrm{BC}$, the rotation of the substituent does not affect the vdW interactions from trans-trans configuration. However, from the changes of $\mathrm{CH}^{\cdots} \mathrm{S}$ to $\mathrm{CH} \cdots \mathrm{HN}$ interaction, cis-trans became more stable than trans-trans configuration. The ciscis configuration had been discussed previously to have highest relative energy. Red-bullet isosurfaces existed in the cis-cis configuration of 1-molecule system, still appeared at the $\mathrm{CH}^{\cdots} \cdot \mathrm{HC}$ pseudo-ring for the dimer, and indicates that the repulsion is due to the ring closure repulsive interactions. As discussed previously, the vdW interaction had shown to affect the stability of the compound. However, only slight vdW interaction is visual due to less interaction between molecules.

(a)

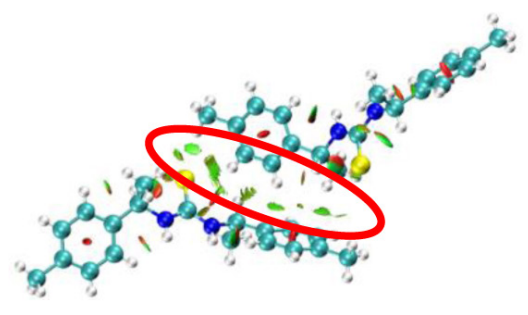

(c)

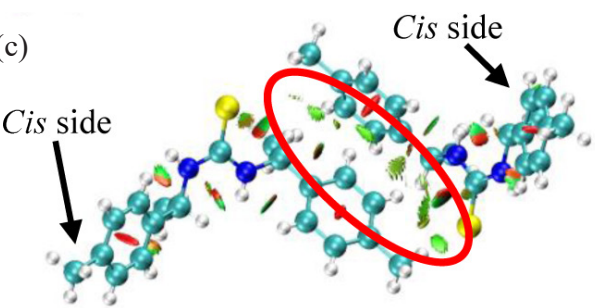

(b)

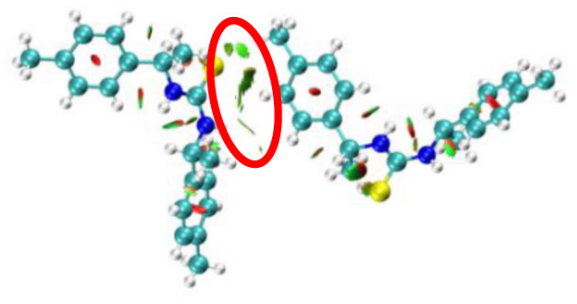

(d)

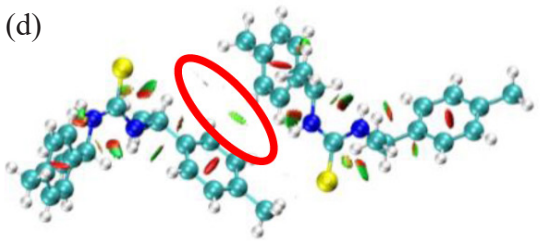

Figure 5: The NCI plot for (a) trans-trans and cis-trans configurations of different substituent couple (b) AC, (c) BC and (d) BD. The red circle on green isosurfaces indicate the van der Waals interaction between molecules. 


\section{CONCLUSION}

The probability of HAMFIL to be a polymorphic compound is studied in this work. The molecules of HAMFIL are rotated along $\mathrm{C}-\mathrm{N}$ bond from trans-trans configuration to cis-trans and cis-cis configurations. The energy analysis results show that the molecules are capable to exist in the cis-trans configuration. Calculations are repeated for a dimer system which results with the same conclusion. NCI isosurface also shows that cis-trans more stable with $\mathrm{CH} \cdots \mathrm{HN}$ interactions compared to $\mathrm{CH} \cdots \mathrm{S}$ interaction in trans-trans.

\section{REFERENCES}

1. Cruz-Cabeza, A. J. \& Bernstein, J. (2014). Conformational polymorphism. Chem. Rev., 114(4), 2170, https://doi.org/10.1021/cr400249d.

2. Price, S. L. (2008). From crystal structure prediction to polymorph prediction: Interpreting the crystal energy landscape. Phys. Chem. Chem. Phys., 10(15), 1996, https://doi.org/10.1039/b719351c.

3. Vázquez, J. et al. (2004). Solvent-free synthesis of chiral N,N'-disubstituted thioureas by 'just-mixing' the reagents. Synth., 12, 1955, https://doi.org/ 10.1055/s-2004-829167.

4. Badri, Z. et al. (2014). Origin of the thermodynamic stability of the polymorph IV of crystalline barbituric acid: Evidence from solid-state NMR and electron density analyses. Cryst. Growth Des., 14(6), 2763, https://doi. org $/ 10.1021 / \operatorname{cg} 401899 q$.

5. Chaudret, R. et al. (2014). Unraveling non-covalent interactions within flexible biomolecules: From electron density topology to gas phase spectroscopy. Phys. Chem. Chem. Phys., 16(21), 9876, https://doi.org/ $10.1039 / \mathrm{c} 3 \mathrm{cp} 52774 \mathrm{c}$.

6. Ang, L. S., Mohamed-Ibrahim, M. I. \& Sulaiman, S. (2016). Polymorphs of tolfenamic acids: Stability analysis using cluster method. Phys. Res. Int., 2016, 5, https://doi.org/10.1155/2016/3537842.

7. Johnson, E. R. et al. (2010). Revealing noncovalent interactions. J. Am. Chem. Soc., 132, 6498, https://doi.org/10.1021/ja100936w. 